\title{
A Case-Study of Bubble Formation Mechanism by Analytical TEM during Evaluation of an Incoming Spin-On-Hardmask at Wafer-Foundries
}

\author{
Wayne W. Zhao* and Frieder Baumann \\ Physical Failure Analysis, Center for Complex Analysis, Characterization Group, Fab8, \\ GLOBALFOUNDRIES, Malta, New York, USA. \\ * Corresponding author: wayne.zhao@globalfoundries.com
}

To increase profit margins at wafer foundries, for high volume manufacturing (HVM) of integrated chips (IC) built by nanometer transistors, it is a must to keep a good balance of the best in class (BIC) quality and the highest yield ratio technically accomplishable while maintaining fast turnaround with costeffective integration schemes [1 7]. Spin-On-Hardmask (SOH) for lithography is one of such matured industrial solutions, which has the benefits of low-cost and high throughput, compared with other approaches, e.g., Amorphous-Carbon-Layer (ACL) and Silicon Oxynitride (SiON) [6]. When and where to apply these schemes, purely depends on specific applications and technical needs for integration.

By transferring desired patterns from masks to surface of real wafers, lithography is the critical process step, and essential for stack up of almost all critical layers in an IC chips HVM, from the front-end-of-line (FEOL), to the middle-of-the-line (MOL), and the back-end-of-the-line (BEOL). Therefore, to scrutinize proactively and tightly control the quality of incoming $\mathrm{SOH}$ from vendors is an unavoidable qualification step. This is because, any tiny imperfection associated with the $\mathrm{SOH}$, eventually, will be translated and manifested into undesired features / defects during various down-stream processes, which will jeopardize the structural integrity of the nanometer-scaled semiconductor device, in terms of yield ratio, device performance, and reliability issues, etc.[7].

In this case-study, we investigated a bubble formation mechanism in one of incoming SOH to be qualified for HVM at wafer foundries. To expedite the evaluation process, various experiments were conducted both with inline non-HVM tools in Fab on short-loop wafers, and offline in characterization labs with expertise specialized in physical failure analysis (PFA) and materials analysis (MA).

Figures. 1a 1c are transmission electron microscopy (TEM) and scanning transmission electron microscopy (STEM) images, and an X-ray energy dispersive spectroscopy (EDS) map, respectively, on the area of interest (AOI), from the SOH with bubbles; while no bubbles seen in a well-controlled $\mathrm{SOH}$ (results not displayed here). Figures. 2a 2c are elemental linescan profiles extracted from the EDS elemental mappings, as illustrated by the arrows (solid, dotted, and double dotted), as shown in Figs.1a 1c right above of each of profiles.

Voids were seen, one in the carbon rich layer (SOH), and the other in OMCTS, octamethylcyclotetrasiloxane (C8H24O4Si4). A bump from the "glue layer" (GL) above NBlok (SiCN) was also noticed. Based on the elemental composition difference, in OMCTS (C8H24O4Si4), vs., tetraethyl orthosilicate (TEOS, $\mathrm{Si}(\mathrm{OC} 2 \mathrm{H} 5) 4)$, $\mathrm{SOH}$ bubble formation and its consequence in transferring to undesired voids during subsequent process was evident. Even if without applying electron energy loss spectroscopy (EELS) quantifications for precise ratio comparison of Si:O:C, the semi-quantitative atomic percentage of Si:O are sufficient, as demonstrated in Fig.2a vs. 2b. Since levels of Silicon and Oxygen were similar in TEOS and the bump adhered from the GL layer, through OMCTS, this indicated a void in 
the OMCTS filled with TEOS with delamination under the OMCTS filled with TEOS as well. That meant, TEOS dipped into the voids existing in OMCTS. A hypothesis is that, this defect creates the void in the new SOH layer (Not seen with original SOH or less extensively). These critical PFA findings provided, (1) solid evidence with great details for decision-making on the business case by upper management of the wafer foundries; (2) guideline for further tighten quality control methodology for such incoming materials, and (3) also a great feedback to the vendor, who is willing to improve their SOH quality, but had a lack of clear directions for corrective actions then, before this in-depth analysis.

References and Acknowledgments:

[1] W Zhao and M Gribelyuk, Microscopy \& Microanalysis 24 (Supplement 1) (2018), p. 1998.

[1] W Zhao et al., Microscopy \& Microanalysis 23 (Supplement 1) (2017), p. 336.

[2] W Zhao and Y Wang, Microscopy \& Microanalysis 23 (Supplement 1) (2017), p. 1490.

[3] B Fu et al., Microscopy \& Microanalysis 23 (Supplement 1) (2017), p. 1456.

[4] W Zhao and M Gribelyuk, Microscopy \& Microanalysis 22 (Supplement 3) (2016), p. 1532.

[5] W Zhao et al., Microscopy \& Microanalysis 20 (Supplement 3) (2014), p. 362.

[6] W-H Wu et al., Proc. of SPIE, Vol. 7140 (2008), p. 71402Q-1.

[7] R Dar et al., Proc. of 44th ISTFA (2018), p. 153.

[8] Thanks go to Jason Behnke, Rebecca Dar, Laurent Dumas, and Corbin Bennett of

GLOBALFOUNDRIES Fab8 for inspiring discussions and technical supports, and Fab8

Management and Legal teams for supporting the publication clearance.
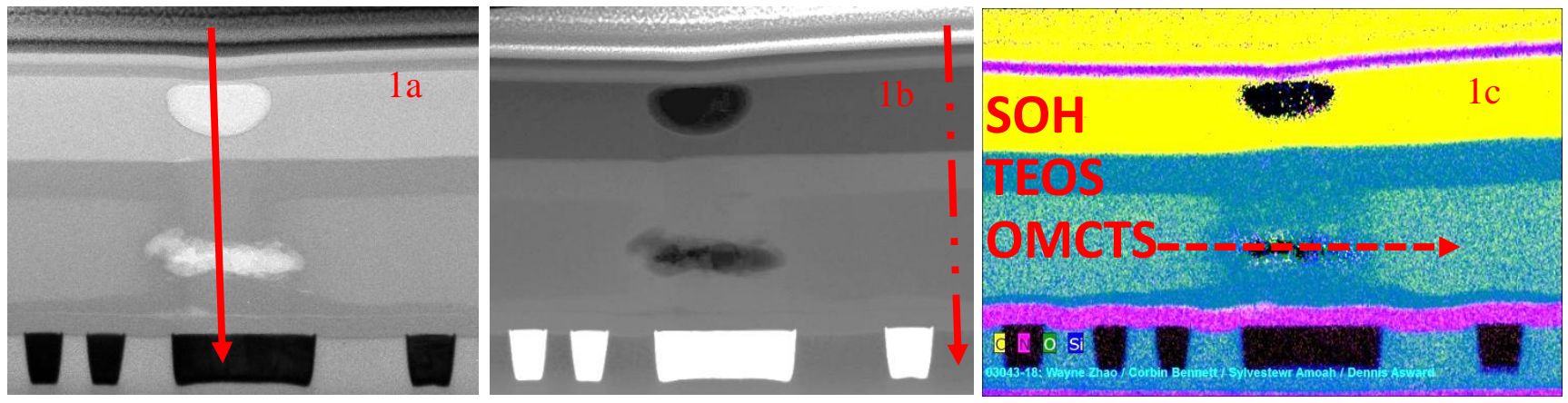

Figure 1. (a) TEM; (b) HAADF-STEM; and (c) EDS mapping, on the SOH bubble.
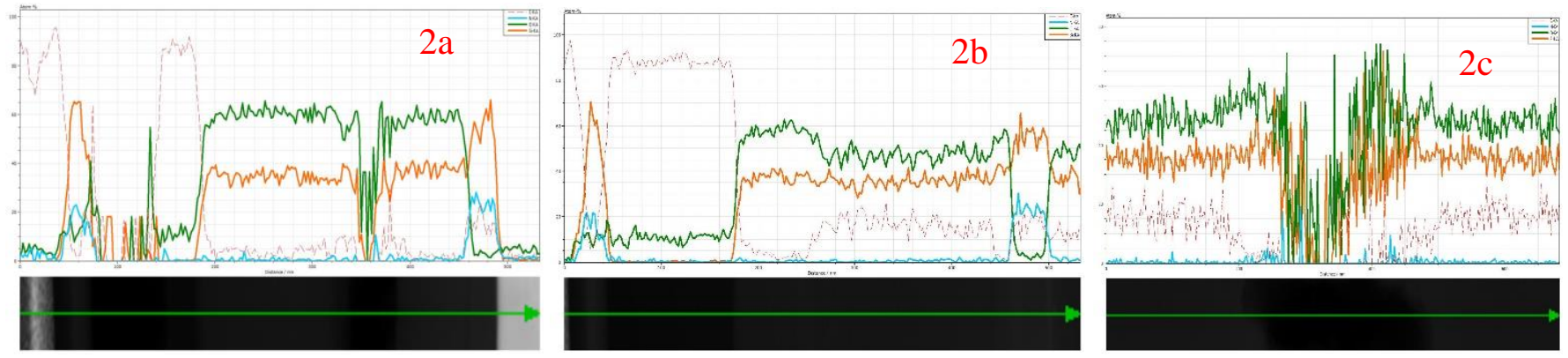

Figure 2. Linescan profiles extracted from the EDS mapping with a path from; (a) top to bottom across the two bubbles; (b) top to bottom across a region without any bubble; and (3) left to right across the bubble in the OMCTS layer. 